\title{
Neural circuit analysis using a novel intersectional split intein-mediated split-Cre recombinase system
}

\author{
Audrey Tze Ting Khoo', Paul Jong Kim', Ho Min Kim²,3 and H. Shawn Je ${ }^{1 *}(\mathbb{D}$
}

\begin{abstract}
The defining features of a neuron are its functional and anatomical connections with thousands of other neurons in the brain. Together, these neurons form functional networks that direct animal behavior. Current approaches that allow the interrogation of specific populations of neurons and neural circuits rely heavily on targeting their gene expression profiles or connectivity. However, these approaches are often unable to delineate specific neuronal populations. Here, we developed a novel intersectional split intein-mediated split-Cre recombinase system that can selectively label specific types of neurons based on their gene expression profiles and structural connectivity. We developed this system by splitting Cre recombinase into two fragments with evolved split inteins and subsequently expressed one fragment under the influence of a cell type-specific promoter in a transgenic animal, and delivered the other fragment via retrograde viral gene transfer. This approach results in the reconstitution of Cre recombinase in only specific population of neurons projecting from a specific brain region or in those of a specific neuronal type. Taken together, our split intein-based split-Cre system will be useful for sophisticated characterization of mammalian brain circuits.
\end{abstract}

Keywords: Split intein, Split-cre recombinase, Circuit mapping, Projection neurons, Retrograde virus, DLX6, GABA, Transgenic mouse

\section{Introduction}

The human brain is arguably the most complex and sophisticated organ made up of intricate networks of neurons connected to each other across different brain regions. These neuronal networks process external stimuli, produce sensory and emotional experiences to generate calculated responses to the environmental stimuli based on intrinsic mechanisms or learned experiences $[21,25,29]$. A fundamental goal in neuroscience therefore is to understand the functions of individual neurons and to delineate how different groups of neurons

\footnotetext{
* Correspondence: shawn.je@duke-nus.edu.sq

${ }^{1}$ Neuroscience and Behavioural Disorders Programme, Duke-National University of Singapore (NUS) Medical School, 8 College Road, Singapore 169857, Singapore

Full list of author information is available at the end of the article
}

function together to orchestrate animal behavior [35]. To achieve this goal, researchers have utilized molecular and genetic tools in model organisms using binary expression systems, such as the Cre-LoxP system in mice and the GAL4-UAS system in Drosophila [2, 31]. Coupled with recent technological advances, including optogenetics to manipulate neuronal activity, voltage- or calcium-sensing proteins to visualize activity, and genetically engineered animals, these binary expression systems have tremendously expanded our knowledge of certain neuronal populations [31, 34].

Although the Cre-LoxP system has been vital in helping us understand neuronal function, single gene expression profile is often insufficient to target specific neuronal populations of interest $[6,10]$. For example, recent results from single-cell RNA sequencing analyses 
indicate high levels of transcriptomic heterogeneity among neurons of the same subtype, even within the same brain subregions [13]. As such, intersectional bipartite systems were developed to target more genetically defined and homogenous populations of neurons. Hirrlinger et al. [6] utilized a Cre-based complementation system by splitting Cre recombinase into two complementation-competent Cre protein fragments, allowing expression of the two split-Cre fragments to be driven by two different promoters. This strategy limited the expression of Cre recombinase to only cells that expressed both fragments of Cre [6]. Similarly, Jullien et al. [9] split Cre recombinase into two complementarycompetent fragments that could be reconstituted via a ligand to achieve better temporal control over the expression of Cre recombinase. Despite their utilities, these split-Cre systems are difficult to implement under conditions that require continuous cellular expression of Cre recombinase as they require exogenous ligands for recombination activity [37]. Here, we present a novel method to reconstitute split-Cre recombinase using split inteins, which are self-catalytic protein elements that facilitate protein trans-splicing reactions $[18,24,27]$, to overcome the need for exogenous ligands to reconstitute Cre recombinase. By using this split intein-mediated split-Cre recombinase system, we aimed to label longrange GABAergic projection neurons that could not be genetically targeted with the current research tools $[8$, $15,20,22]$. By simply expressing one split-Cre fragment in the neurons of the GABAergic lineage and delivering the other fragment via retrograde viral gene transfer, we were able to constitute Cre activity in only long-range GABAergic neurons that projected their axons from the central amygdala (CeA) to the dorsal striatum (DS).

\section{Results}

We split Cre recombinase into two fragments, NCre (aa1-59) and CCre (aa60-343) [10], and attached an Nor C-intein of the evolved split intein Npu37, which can express and trans-splice efficiently in mammalian cells [18], to the C-terminal sequence of NCre and the $\mathrm{N}$ terminal sequence of CCre respectively. This resulted in two fusion genes: NCre-IntN and IntC-CCre (Fig. 1a). The transcription and translation of the NCre-IntN and IntC-CCre fusion genes lead to the binding and autocatalytic trans-splicing of the split inteins. This reaction then ligates NCre and CCre together via peptide bonds to form functional Cre recombinase with additional peptide sequences (KGCFNKEDGS from IntC and GFL from IntN) [26]. Based on our 3D modeling, the additionally incorporated peptide sequences do not occlude the active DNA-binding site of the reconstituted Cre recombinase (Fig. 1b). To test whether split inteinmediated trans-splicing could occur in mammalian cells under physiological conditions, we expressed epitopetagged NCre-IntN and IntC-CCre constructs in HEK293T cells, and subjected the resulting lysates to western blot analysis (Fig. 1c and d). When either NCreIntN or IntC-CCre was transfected into HEK293T cells alone, only a single $49 \mathrm{kD}$ band or a $37 \mathrm{kD}$ band was observed by western blot using specific antibodies against either HA or FLAG respectively (Fig. 1d). However, when both NCre-IntN and IntC-CCre were transfected, we observed a band of higher molecular weight $(67 \mathrm{kD})$ detectable by both HA and FLAG specific antibodies, indicating successful split intein-mediated trans-splicing reaction under physiological conditions (Fig. 1d).

Next, to validate whether the reconstituted Cre recombinase via trans-splicing of split inteins was functional, we attached fluorescent tags, mTagBFP2 and Cerulean to NCre-IntN and IntC-CCre respectively, to visualize the cellular expression of these constructs in HEK293T cells (Fig. 1e). We reasoned that the expression of these split-Cre constructs together with a LoxP-stop-LoxP DsRed reporter cDNA would easily reflect the activity and efficiency of split-Cre recombination through the presence of DsRed fluorescence in cells (Fig. 1e). As expected, we did not observe any DsRed fluorescence when either NCre-IntN or IntC-CCre was transfected alone. However, when both NCre-IntN and IntC-CCre were cotransfected, we observed strong DsRed signals, which was similar to cells transfected with the fulllength Cre recombinase cDNA and reporter (Fig. 1f). We also observed that approximately $99 \%$ of the cells expressing both NCre-IntN and IntC-CCre exhibited DsRed fluorescence (Fig. 1f). Alternatively, we performed a luciferase assay as a readout for Cre-mediated recombination activity [11] by transfecting either NCre-IntN or IntC-CCre alone, or together into HEK293T cells expressing a LoxP-stop-LoxP luciferase cDNA construct (Fig. 2a). Twenty-four hours after transfection, we detected luminescence in only the cells cotransfected with both NCre-IntN and IntC-CCre $(p<0.001$; Fig. $2 \mathrm{~b})$. Importantly, we observed that the Cre activities of reconstituted split intein split-Cre recombinase were comparable to that of native Cre recombinase $(p=0.798$, no statistical significance; Fig. 2b [7]).

To test if the split-Cre system is functional in the in vivo mammalian brain, we delivered NCre-IntN and IntC-CCre, Cre recombinase, or GFP only constructs into the lateral ventricles of LoxP-stop-LoxP-TdTomato reporter mice on embryonic day 16.5 (E16.5) via in utero electroporation [36] (Fig. 3a). We did not observe any TdTomato expression when we electroporated GFP only or IntC-CCre fragment only (Fig. 3b). However, we observed strong TdTomato fluorescence in neurons when we electroporated both NCre-IntN and IntC-CCre constructs, and the fluorescence signal as well as a number 
A

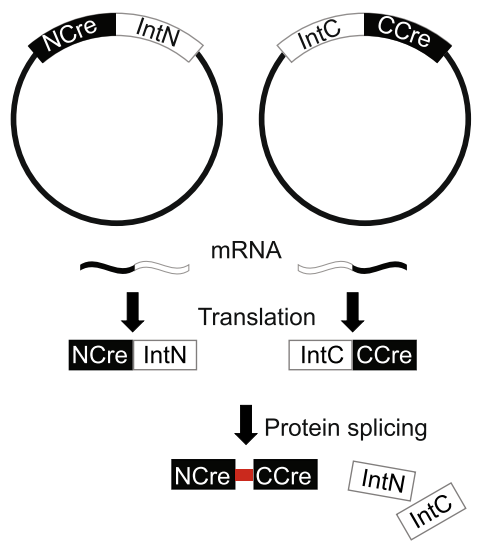

C

NCre (49 kD):

\begin{tabular}{|l|l|l|l|l|}
\hline NLS & HA & Cerulean NCre & IntN \\
\hline
\end{tabular}

CCre (37 kD):

IntC CCre FLAG

NCre + CCre (67 kD):

\begin{tabular}{|l|l|l|l|l|}
\hline NLS & HA & Cerulean NCre & CCre & FLAG \\
\hline
\end{tabular}

E
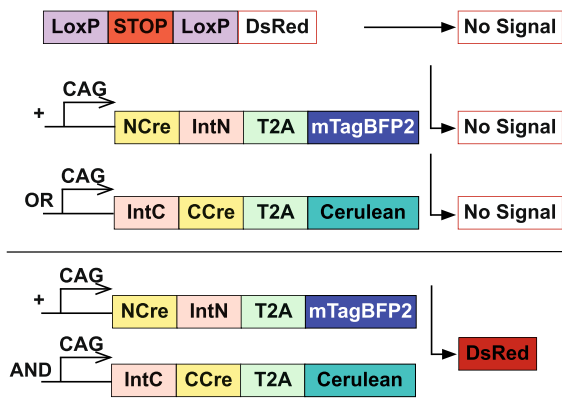

B

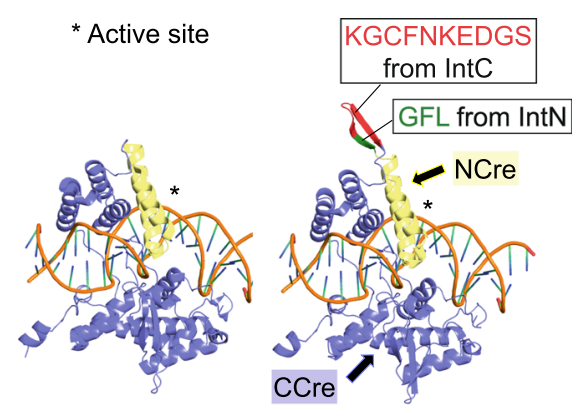

Cre recombinase

D

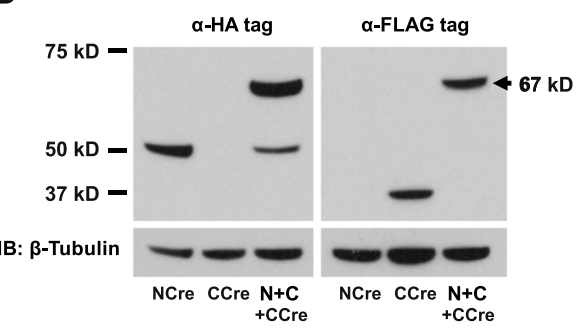

$\mathbf{F}$

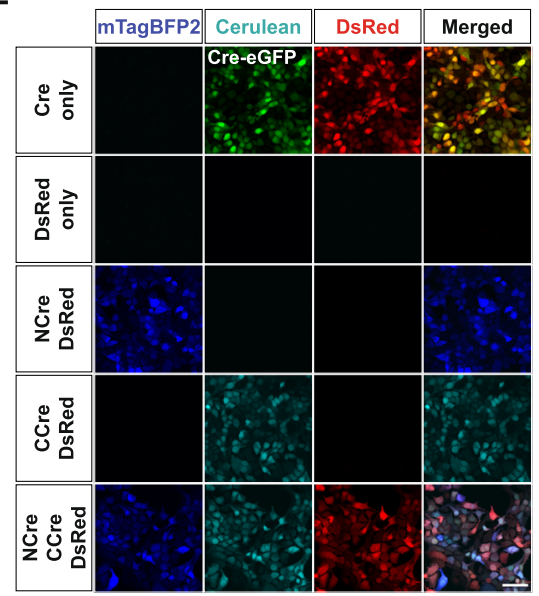

Fig. 1 Design of the split intein-mediated split-Cre recombinase system. a Schematic depicting the autocatalytic split intein-mediated transsplicing reaction to reconstitute Cre recombinase. Split inteins associate to fuse NCre and CCre with a peptide bond. b Structural comparison of original Cre recombinase-LoxP DNA complex (left, PDB ID: 1NZB [5] with a model for split intein-mediated split-Cre recombinase-LoxP DNA complex (right). The structure of split inteins from IntN (GFL) and IntC (KGCFNKEDGS), NCre and CCre are colored as green, red, yellow and blue respectively. Conserved active site are marked with an asterisk. c Schematic demonstrating the predicted size of the protein when NCre-IntN tagged with HA (49 kD) or IntC-CCre tagged with FLAG (37 kD) are transfected alone. A $67 \mathrm{kD}$ reconstituted product is expected when both NCre-IntN and IntC-CCre are cotransfected. $\mathbf{d}$ Western blot analysis showing that when NCre-IntN is transfected alone, a $37 \mathrm{kD}$ protein band was observed when western blotting was performed using a specific antibody against HA. When IntC-CCre was transfected alone, a 49 kD protein band was observed when western blotting was performed using a specific antibody against FLAG. When NCre-IntN and IntC-CCre were cotransfected, a $67 \mathrm{kD}$ protein band with a higher molecular weight was observed when western blotting was performed using a specific antibody against HA or FLAG. These results indicated that the reconstituted $67 \mathrm{kD}$ product comprised of components from both NCre-IntN and IntC-CCre. e Design of the in vitro reporter assay. HEK293T cells were transfected with NCre-IntN or IntC-CCre alone or together with a LoxP-StopLoxP-DsRed reporter. $\mathbf{f}$ When NCre-IntN or IntC-CCre were transfected alone, no DsRed expression was observed. When NCre-IntN and IntC-CCre were cotransfected along with the reporter in HEK293T cells, we observed strong DsRed expression. Scale bar, $50 \mu \mathrm{m}$ 
A

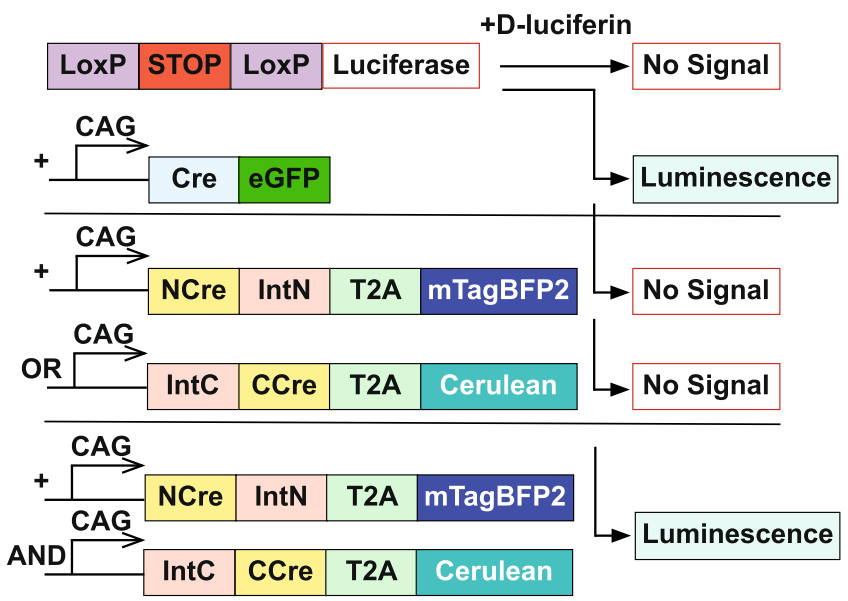

B
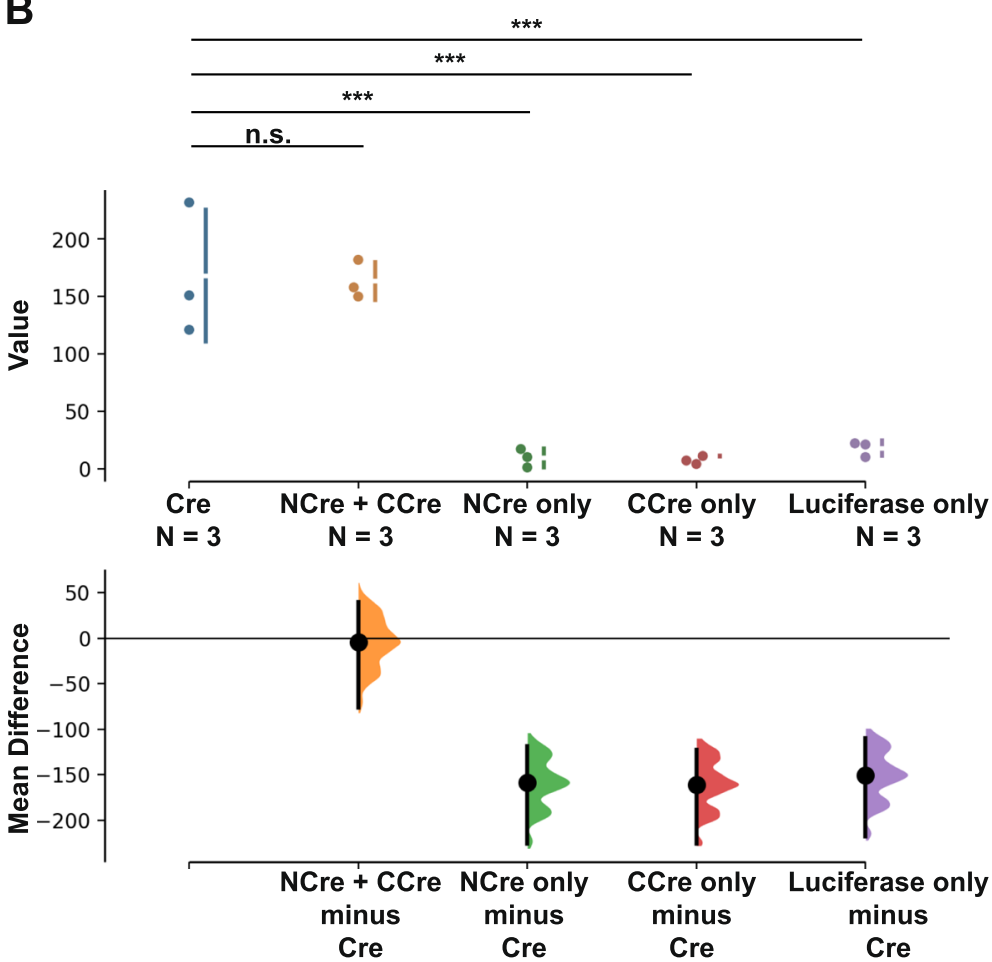

Fig. 2 Design of luciferase assay. HEK293T cells were transfected with Cre recombinase, NCre-IntN or IntC-CCre alone, or together, with a LoxP-stop-LoxPLuciferase reporter. a Schematic showing the experimental design for transfecting cDNA constructs in HEK293T cells. b The Cre activity is defined by the amount of luminescence detected in the luciferase assay. The mean difference for 4 comparisons against the shared control Cre recombinase are shown in the above Cumming estimation plot. The raw data is plotted on the upper axes. On the lower axes, mean differences are plotted as bootstrap sampling distributions. Each mean difference is depicted as a dot. Each 95\% confidence interval is indicated by the ends of the vertical error bars. Importantly, we observed similar levels of Cre activity for both the native Cre recombinase and the reconstituted split-Cre recombinase. ${ }^{*} p<0.05$, ${ }^{* *} p<0.01$, ${ }^{* * *} p<0.001$

of expressed neurons were similar to electroporation of native Cre recombinase (Fig. 3b). Taken together, these data showed that the reconstituted split intein-mediated split-Cre recombinase is functional in both in vitro and in vivo in mammalian brains.

To target long-range GABAergic projection neurons [30], we generated a transgenic mouse that expressed IntC-CCreIRES-eGFP under the control of an endogenous $D l \times 6$ promoter known to be expressed exclusively in forebrain GABAergic neurons [1, 4, 14, 33] (Fig. 3c and d). Next, the resulting $D l x 6$-CCre-IRES-eGFP mice were further crossed with Rosa26-LoxP-stop-LoxP-TdTomato reporter mice [19] (Fig. 3d). Using immunohistochemistry, we confirmed that IntC-CCre expression was restricted to GABAergic neuronal populations (Fig. 3e and f). A total of $83.1 \%$ of the IntCCCre neurons, indicated by green fluorescence, expressed 
A

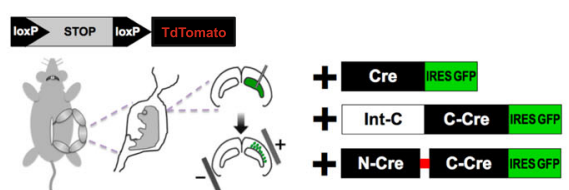

in utero electroporation at E16.5

C

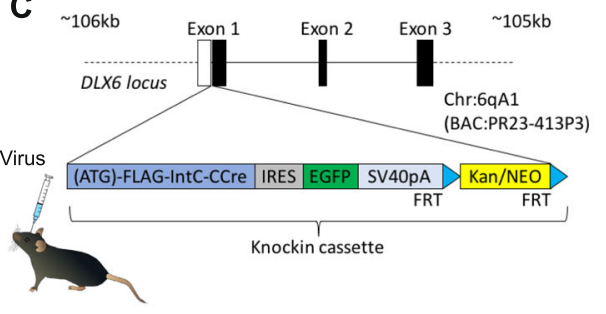

D

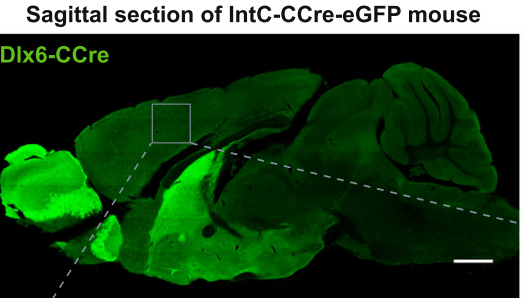

B

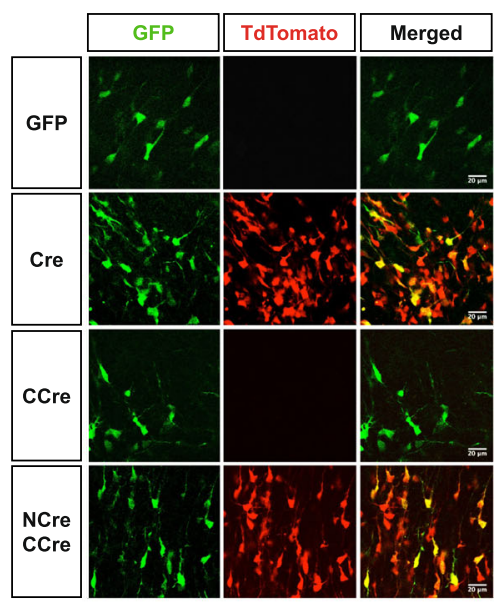

$\mathbf{E}$

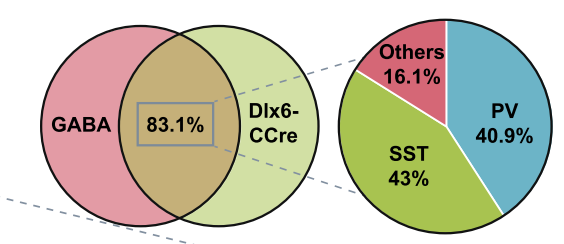

$\mathbf{F}$

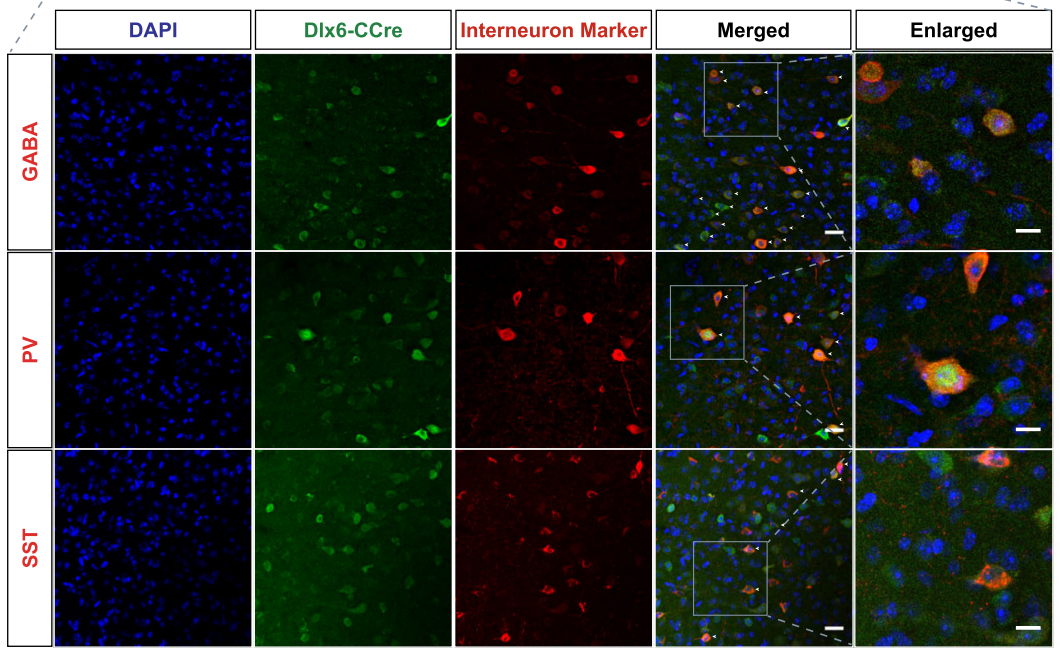

Fig. 3 In vivo validation of the split intein-mediated split-Cre recombinase system. a Schematic showing the experimental design for electroporating constructs into LoxP-Stop-LoxP-TdTomato E16.5 embryos. b Strong TdTomato expression was observed with native Cre recombinase or when both NCre-IntN and IntC-CCre constructs were electroporated. No TdTomato was observed when only IntC-CCre was electroporated alone. This demonstrated that Cre-mediated recombination occurs only when both NCre and CCre are present. c Schematic diagram of BAC transgenic mice expressing IntC-CCre in forebrain GABAergic neurons. The resultant BAC transgenic mouse was then further crossed with the LoxP-Stop-LoxP-TdTomato reporter line mouse. Scale bar, $20 \mu \mathrm{m}$. d Sagittal section from a DLX-CCre-IRES-eGFP mouse showing that IntC-CCre-positive cells were predominantly found in the forebrain. Scale bar, $1 \mathrm{~mm}$. e Venn diagram showing that 83.1\% of DLX6-CCre neurons were GABA-positive. Of the CCre+/GABA+ neurons, 36.5\% were PV and 41\% were SST. f Immunohistochemical analyses showing DLX6CCre-IRES-eGFP brain sections stained using specific antibodies against GABA, PV and SST. Scale bars, $20 \mu \mathrm{m}$ (merged) and $10 \mu \mathrm{m}$ (enlarged).

GABA, and 40.9 and $43 \%$ of these neurons expressed PV and SST respectively (Fig. 3e and f).

First, we validated the reconstitution of split-Cre in our Dlx6-CCre-IRES-eGFP mice. We generated lentiviral vectors encoding either mTagBFP2 or NCre-IntN-mTagBFP2.
We injected mTagBFP2 lentiviral particles as a control into one hemisphere of the striatum and injected NCre-IntNmTagBFP2 particles into the other (Fig. 4a). While we did not observe any TdTomato expression in the hemisphere injected with mTagBFP2, robust TdTomato expression was 


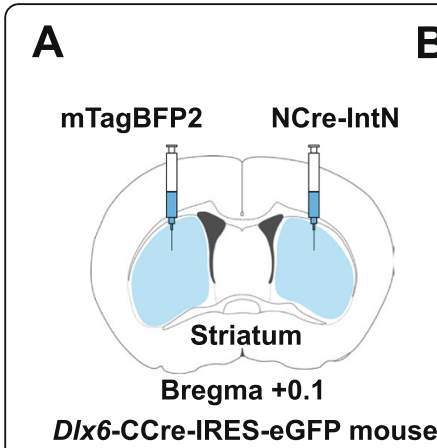

B

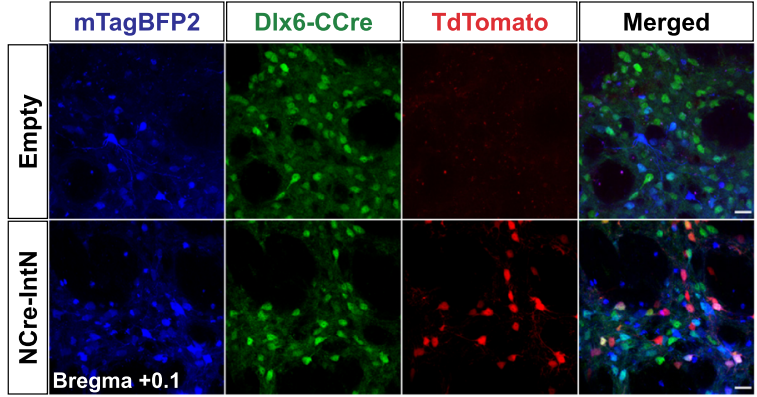

C

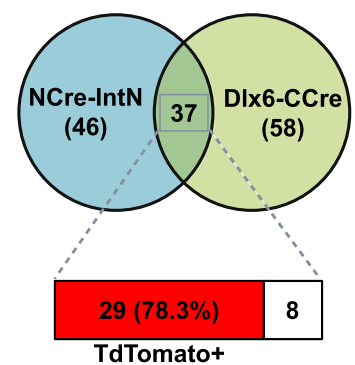

$\mathbf{F}$

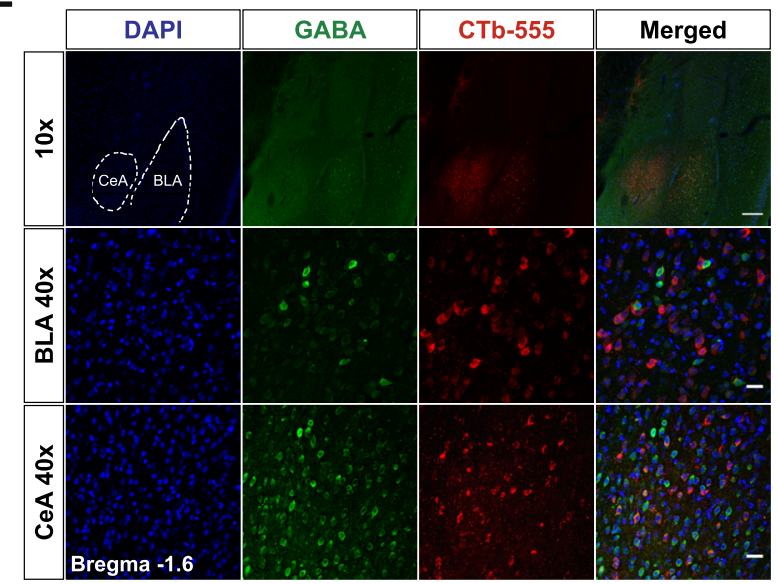

Bregma -1.6

E

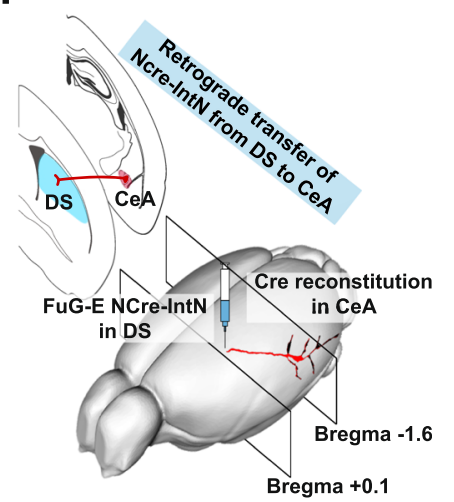

DIx6-CCre-IRES-eGFP:: LoxP-stop-LoxP-TdTomato mouse
Injection Site of CTb-555 in wildtype mouse

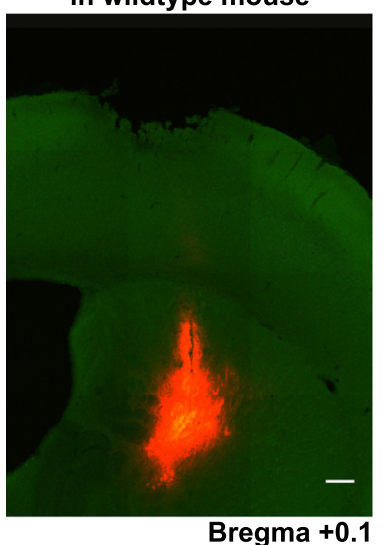

G

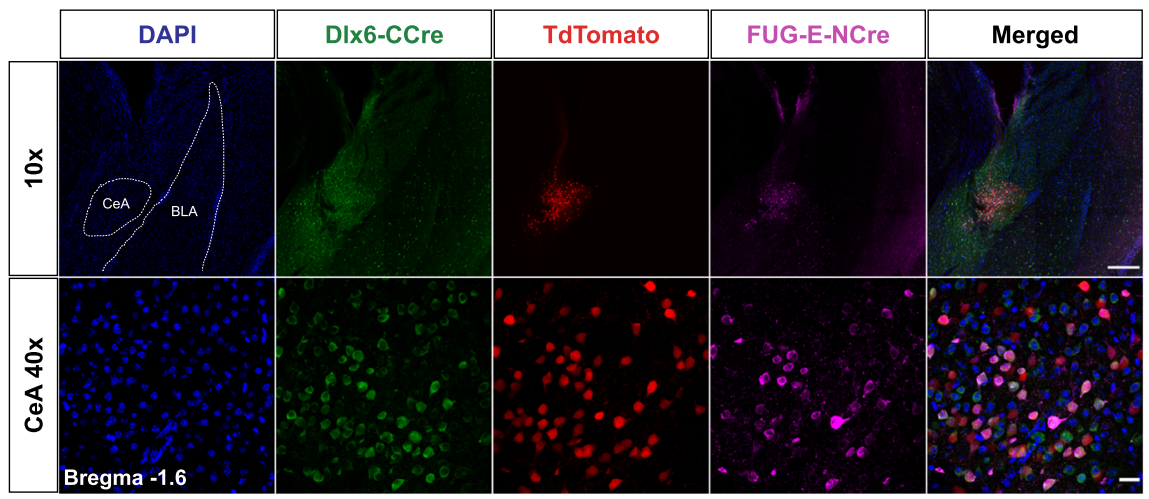

H

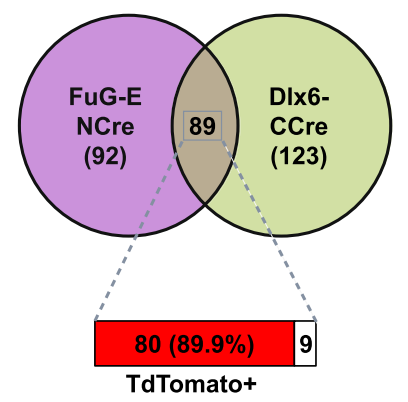

Fig. 4 Targeting long-range GABAergic neurons using a DLX6-CCre-IRES-eGFP mouse and virus. a Schematic of stereotaxic injection of lentiviral particles to observe recombination of Cre recombinase in DLX6-CCre-IRES-eGFP mice. The mTagBFP2-only lentiviral particles were injected into one hemisphere or the striatum, while the NCre-IntN-carrying lentiviral particles were injected into the other hemisphere. $\mathbf{b}$ In the hemisphere injected with the mTagBFP2-only lentiviral particles, no TdTomato signal was observed. However, in the hemisphere injected with NCre-IntNcarrying lentiviral particles, a TdTomato signal was observed, indicating the presence of functional Cre recombinase. Scale bar, $25 \mu \mathrm{m}$. c Venn diagram showing the percentage of cells expressing TdTomato. $\mathbf{d}$ Microscopic image showing the injection site of CTb-555 in the DS of wildtype mice. Scale bar, $200 \mu \mathrm{m}$. e CTb-555-labeled cells were observed in both the CeA and BLA. However, immunohistochemical analysis illustrated that only labeled cells in the CeA were GABAergic, while those in the BLA were not. Scale bars, $200 \mu \mathrm{m}$ (10x) and $20 \mu \mathrm{m}$ (40x). f Schematic diagram showing projection from CeA to DS. The FuG-E-NCre virus was injected into the DS of D/x6-CCre-IRES-eGFP::LoxP-stop-LoxP-TdTomato mice, which was retrogradely transferred to the CeA. Cre recombination is expected to occur in GABAergic neurons in the CeA that project to the DS. g When FuG-E-NCre pseudotyped lentiviral particles were injected into the Dlx6-CCre-IRES-eGFP::LoxP-stop-LoxP-TdTomato mice, TdTomato cells were observed in the CeA. Scale bars, $200 \mu \mathrm{m}$ (10x) and $20 \mu \mathrm{m}$ (40x). $\mathbf{h}$ Venn diagram showing the percentage of cells expressing TdTomato in the CeA 
evident in the hemisphere injected with NCre-IntNmTagBFP2 (Fig. 4b). These results indicated that selective reconstitution of recombinatory Cre function occurred only when both NCre-IntN and IntC-CCre were expressed. Of the 37 neurons expressing both NCreIntN and IntC-CCre, 78.3\% exhibited a strong TdTomato fluorescence signal (Fig. 4c).

To selectively target long-range GABAergic projection neurons, we first validated the presence of these types of neurons that were found to project from the central amygdala (CeA) to the dorsal striatum (DS) [16] by injecting cholera toxin subunit B fused to Alexa Fluor 555 (CTb-555) into the DS of wild-type mice (Fig. 4d) [32]. We observed CTb-555 labeling in both the CeA and basolateral amygdala (BLA) (Fig. 4e). Immunohistochemistry analyses showed that only CTb-labeled neurons in the CeA were GABA-positive, whereas the $\mathrm{CTb}$ labeled neurons in the BLA were not (Fig. 4e). This confirmed that only the projections from the CeA to the DS were from GABAergic neurons, as reported by Lingawi and Balleine [16]. Next, we generated retrograde lentiviral particles carrying NCre-IntN by substituting the vesicular stomatitis virus glycoprotein (VSV-G) with FuG-E, a pseudotyped lentiviral envelope where its fusion glycoprotein comprises of VSV-G and the rabies virus glycoprotein (RV-G) [12]. This allows NCre-IntN to be retrogradely transferred from the axon terminating in the DS back to the cell body of neurons in the CeA (Fig. 4f). Injection of the FuG-E-NCre-IntN viral particles into the DS of the Dlx6-CCre-IRES-eGFP::LoxPstop-LoxP-TdTomato mice resulted in TdTomatoexpressing neurons within the CeA $(89.9 \%$ NCre- and CCre-expressing neurons expressed TdTomato) (Fig. 4g and $\mathrm{h}$ ). While we observed NCre-IntN expression in some BLA neurons as the BLA also projects to the DS, we did not observe a TdTomato signal, indicating that Cre reconstitution was highly specific (Fig. 4g).

\section{Discussion}

Here, we developed a split-Cre system based on split intein trans-splicing mechanisms. By generating a transgenic mouse model that expresses IntC-CCre in forebrain GABAergic neurons and NCre-IntN expressing retrograde viral particles, we could selectively target long-range GABAergic neurons projecting from the CeA to the DS (Fig. 4g). In the Dlx6-CCre-IRES-eGFP mouse generated, we noted only $83.1 \%$ of the IntC-CCre neurons were GABA-positive. This discrepancy could have arisen from our conservative approach in quantifying GABA-positive signals (2 standard deviations away from background signal) and the relatively thick $40 \mu \mathrm{m}$ tissue preparation that could lead to poor antibody penetration and underestimation of GABA-positive neurons. We are still puzzled by the slightly lower recombination efficiency of our split-Cre system upon viral delivery schemes in vivo (approximately 80\%, compared to 99\% upon transfection; Fig. 4c and h), which could simply be due to the low titer $(3 \mathrm{E} 8 \mathrm{IU} / \mathrm{mL}$ ) of our lentiviral particles. However, this result also indicates the selectivity and stringency of our split-Cre recombinase, since germline recombination and transient unwanted expression of Cre recombinase have been reported in even wellestablished transgenic mouse lines [28]. Given that we can genetically target long-range GABAergic neurons with high specificity, we can further investigate the functions of long-range GABAergic projection neurons during active behavior using existing Cre-dependent optoand chemogenetic tools. In addition, our approach can be used to understand whether the projection patterns of a neuron correlates with its gene expression profile using RNA sequencing. Our approach should be able overcome the limitation of having few virally packageable, tissue-specific promoters [23].

Taken together, our combinatory split intein-mediated split-Cre system allows us to limit Cre recombinase expression to specific subsets of neurons by means of its gene expression profile and anatomical connections, enabling the systematic study of specific types of neurons in the mammalian brain.

\section{Methods}

Computer simulation of split-intein split-Cre recombinase product

Model for split-intein mediated split-Cre recombinaseLoxP DNA complex was calculated using automated modelling mode in the SWISS model server (http:// swissmodel.expasy.org/), based on the crystal structure of Cre-LoxP synaptic complex (1NZB) as a template [5]. Split inteins is expected to form stable $\beta$-turn structure without any substantial structural changes in the original Cre recombinase. Figures for the structure were prepared using PyMol software [3].

\section{Construction of NCre-IntN and IntC-CCre}

NCre and CCre were generated from the pCAG-Cre:GFP, a gift from Connie Cepko (Addgene plasmid \#13776; https:/www.addgene.org/13776/; RRID:Addgene_13,776) using the KAPA HiFi PCR kit (Roche Sequencing). IntN and IntC were amplified from the Npu DnaE inteins with site directed mutagenesis according to Lockless and Muir [18]. All plasmids were subcloned into a pCDH expression vector (System Biosciences), assembled using Gibson Assembly (New England Biolabs) and verified by Sanger DNA sequencing.

\section{Western blot analyses}

HEK293T cells in 12-well culture plates were transfected with $1 \mu \mathrm{g}$ of each construct and Lipofectamine 2000 
(Thermo Fisher Scientific) for $18 \mathrm{~h}$. Cells were supplied with fresh media after transfection and grown for another 2 days. Cells were mechanically lysed in RIPA buffer containing phosphatase and protease inhibitors. The proteins were separated by SDS-PAGE under reducing conditions and transferred to polyvinyl difluoride (PVDF) membranes (Millipore, Billerica, MA). Antibody incubations were performed in 3\% BSA in TBS buffer using the following antibodies: anti-HA, 1:1000 (MilliporeSigma); anti-FLAG, 1:1000 (MilliporeSigma); anti- $\beta$ Tubulin, 1:1000 (Covance).

\section{HEK293T in vitro reporter assay}

HEK293T cells were seeded into 12-well culture plates containing coverslips treated with poly-L-lysine and transfected the next day with $1 \mu \mathrm{g}$ of each construct using Lipofectamine 2000 (ThermoFisher Scientific) for $18 \mathrm{~h}$. The cells were supplied with fresh media after transfection and subsequently fixed with $4 \%(\mathrm{w} / \mathrm{v})$ paraformaldehyde in PB 24h later. The coverslips were mounted onto microscope slides with FluorSave mounting medium (Calbiochem) and imaged with the Zeiss LSM780 confocal microscope (Zeiss Microscopy, Munich, Germany) using the 40x oil objective lens.

\section{HEK293T luciferase assay}

HEK293T cells were seeded into 96-well culture plates and transfected with $1 \mu \mathrm{g}$ of each constructed the next day. $24 \mathrm{~h}$ post transfected, we washed the cells with PBS and treated them with lysis buffer (Pierce Firefly Luciferase Glow Assay Kit, Thermo Fisher Scientific) for 20 min. The cell lysate was then collected and spun down to remove cell debris. $15 \mu \mathrm{l}$ of the cell lysate was transferred into a 96-well black polysterene plate (Corning Costar). $45 \mu \mathrm{l}$ of the working buffer (D-luciferin and the Firefly Glow Assay buffer according to manufacturer's instructions, Pierce Firefly Luciferase Glow Assay Kit, Thermo Fisher Scientific) was added to the cell lysate and we waited $10 \mathrm{~min}$ before acquiring signals using a microplate reader (Tecan, Männedorf, Switzerland).

\section{Animals}

Animals were housed in a specific pathogen-free facility maintained below $22{ }^{\circ} \mathrm{C}$ and $55 \%$ humidity under a $12 \mathrm{~h}$ light-dark cycle (lights on at $0700 \mathrm{~h}$ ) with ad libitum access to food and water. All experimental procedures were conducted in accordance with guidelines for the care and use of laboratory animals for scientific purposes with approved protocols from the Institution Animal Care and Use Committee of SingHealth Research (Protocol number: 2014/SHS/921).

\section{In utero electroporation}

Floxed Tdtomato transgenic females were time mated. On embryonic day 16.5 (E16.5), ex vivo electroporation and organotypic brain slice culture was performed as described elsewhere [17]. A GFP expression plasmid was used as a negative control, and a native Cre recombinase plasmid was used as a positive control. For the experiments, embryos were electroporated either with CCre plasmid only or with both NCre and CCre plasmids. For image acquisition, on day 3 of culture, the organotypic slice inserts were transferred to a $50 \mathrm{~mm}$ petri dish containing culture medium and imaged using an inverted long distance fluorescent microscope.

\section{Generation of DLX6-IntC-CCre-eGFP mice}

We used the bacterial artificial chromosome (BAC) transgenic approach to generate the animals that express IntC-CCre under the DLX6 promoter. In this experiment, we constructed a knock-in cassette containing a FLAG-tagged (at the N-terminal of) IntCCCre coding sequence followed by an IRES-eGFP cassette. A SV40 polyadenylation signal sequence was placed at the $3^{\prime}$ end of the cassette to stabilize the transcript. A FRT flanked Kan/Neo selection cassette was attached to the $3^{\prime}$ end of the IntC-CCre cassette to facilitate screening of the recombinant clones. We used recombineering technology to integrate the IntC-CCre expression cassette into the $\mathrm{BAC}$ clones containing the Dlx gene locus. In the recombinant BAC clones, the translation start codon ATG of FLAG-IntC-CCre is fused to the ATG of the endogenous Dlx gene. After deletion of the selection cassette by expression of Flp, the resultant BAC clones containing the IntC-CCre cassette were used to create the transgenic mice by standard pronuclear injection.

\section{Virus preparation}

HEK293T cells were cultured in T75 flasks and transfected with hSyn.NCre-IntN.T2A.mTagBFP2, envelope and VSV-G or FuG-E packaging plasmid. pCAGGSFuG-E was a gift from Dr. Kazuto Kobayashi (Addgene plasmid \#67509; http://n2t.net/addgene:67509; RRID: Addgene_67,509). $18 \mathrm{~h}$ after transfection, we supplied fresh medium and the cells were further incubated for $48 \mathrm{~h}$. The medium was collected and filtered through a $0.45 \mu \mathrm{m}$ Minisart syringe filter (Sartorius Stedim, Goettingen, Germany). Viral vector particles were pelleted via ultracentrifugation at $25,000 \mathrm{~g}$ for $2 \mathrm{~h}$. Supernatant was subsequently removed and the pellet was resuspended in sterile PBS overnight. Virus titre was quantified using the qPCR Lentivirus Titration Kit according to 
manufacturer's directions (Applied Biological Materials, British Columbia, Canada).

\section{Stereotaxic injection of virus and cholera toxin subunit B} Mice were anesthetized with $2 \%$ isoflurane in oxygen at a flow rate of $0.5 \mathrm{~L} / \mathrm{min}$. Mice were placed in a stereotaxic apparatus to receive bilateral infusions of the virus or $0.5 \%(\mathrm{w} / \mathrm{v})$ cholera toxin subunit B (CTb) Alexa Fluor 555 Conjugate (Invitrogen) into the dorsal striatum (+ $0.1 \mathrm{~mm}$ antero-posterior, $2.0 \mathrm{~mm}$ mediolateral, $2.7 \mathrm{~mm}$ dorsoventral); coordinates relative to bregma (Paxinos and Watson, 2007). 1ul of virus or $0.1 \mu \mathrm{l}$ of CTb-555 was infused per hemisphere with a 30 -gauge needle (Hamilton, United States) over $5 \mathrm{~min}$ and $0.5 \mathrm{~min}$ respectively using a programmable infusion pump (Hamilton, United States). The needle was left in the place for $5 \mathrm{~min}$ to allow diffusion of the virus or CTb before removal at $0.1 \mathrm{~mm} / \mathrm{sec}$. Following surgery, mice received $5 \mathrm{mg} / \mathrm{kg}$ carprofen intraperitoneally. Mice were sacrificed 28 days or 7 days following virus or CTb injection respectively.

\section{Perfusion and immunohistochemical analyses}

Mice were anesthetized with isoflurane and transcardially perfused with ice-cold PBS, followed by $4 \%$ (w/ v) paraformaldehyde (PFA) in 0.1 M PB. Extracted brains were submerged in PFA for an hour before transferring to $30 \%(\mathrm{w} / \mathrm{v})$ sucrose in $0.1 \mathrm{MPB}$ for cryoprotection. After $48 \mathrm{~h}$, brain were sectioned at $40 \mu \mathrm{m}$ and stored in PBS-Azide. Sections were first permeabilized in $0.2 \%$ Triton-X100 in PBS (30 min, RT) and later incubated in (i) a blocking solution of in PBS containing 5\% donkey serum, 3\% bovine serum albumin and $0.2 \%$ Triton-X100 (1 h, RT); (ii) the same solution containing the primary antibody overnight at $4{ }^{\circ} \mathrm{C}$ (anti-tRFP, 1:1000, Evrogen; antiGFP, 1:1000, Abcam; anti-GABA, 1:2000, MilliporeSigma; anti-parvalbumin, 1:2000, Abcam; antisomatostatin, 1:1000, Peninsula Laboratories) (iii) the same solution containing a 1:500 dilution of secondary antibody (Alexa Fluor, ThermoFisher; $2 \mathrm{~h}, \mathrm{RT}$ ). All sections were then stained with DAPI (1:5000 diluted in $0.2 \%$ Triton X-100 in PBS, Sigma-Aldrich), washed in $0.2 \%$ Trixton-X100 in PBS and mounted with FluorSave (Merck Millipore). Images of the immunostained sections were acquired using the widefield (Nikon Instruments, Tokyo, Japan) or confocal microscope (Zeiss Microscopy, Munich, Germany).

\section{Statistical analyses}

Data were analysed and plotted using Estimation stats/ plots [7]. $p<0.05$ was considered statistically significant.

\section{Supplementary information}

Supplementary information accompanies this paper at https://doi.org/10. 1186/s13041-020-00640-2.

Additional file 1: Figure S1. Immunohistochemistry performed in 6 month old D/x6-CCre-IRES-eGFP mice. S1(A-C) Microscope images of immunohistochemistry performed in $40 \mu \mathrm{m}$ thick coronal sections using antibodies against Calcium/calmodulin-dependent protein kinase II (CaMKII), excitatory neuron marker (A), choline acetyltransferase (ChAT), cholinergic neuron marker (B) and glial fibrillary acidic protein (GFAP), astrocyte marker (C). Arrows indicate Dlx6-CCre-positive neurons based on GFP fluorescence do not overlap with either CaMKII, ChAT or GFAP signals. Scale bars: $25 \mu \mathrm{m}$ in panel A, $50 \mu \mathrm{m}$ in panels B and $C$.

Additional file 2: Figure S2. Brain sections of 6 month old D/x6-CCreIRES-eGFP mice showing normal gross brain structure. S2(A) A sagittal brain section of D/x6-CCre-IRES-eGFP mice stained with DAPI. Scale bar: 1 $\mathrm{mm}$. S2(B) Coronal forebrain sections of D/x6-CCre-IRES-eGFP mice taken approximately $1 \mathrm{~mm}$ apart, stained with DAPI. Scale bar: $1 \mathrm{~mm}$.

Additional file 3: Figure S3. Quantitative analyses of a 10 min open field test performed using 1 year old D/x6-negative::Rosa26-LoxP-StopLoxP-TdTomato (littermate control [DIx6- RTM]), and D/x6-CCre-IRESeGFP::Rosa26-LoxP-Stop-LoxP-TdTomato (Dlx6+ RTM). S3(A) Total distance travelled in open field chamber (cm), (B) Number of movements, (C) Number of stereotypy, which is the amount of repetitive movements, includeing grooming behaviour, (D) Time spent in center (s). We noted statistically non-significant differences (Mann-Whitney test) between Dlx6RTM and Dlx6+ RTM in locomotor activity ( $p>0.9999)$, movements ( $p=$ $0.9143)$, stereotypy $(p=0.2571)$ and time in center $(p=0.2571)$.

\section{Abbreviations}

BAC: Bacterial artificial chromosomes; BLA: Basolateral amygdala; CCre: Cterminal Cre fragment; CeA: Central amygdala; CTb: Cholera toxin subunit B; DNA: Deoxyribonucleic acid; DS: Dorsal striatum; FuG-E: Fusion glycoprotein type E; GABA: Gamma aminobutyric acid; HEK293T: Human embryonic kidney 293 cells expressing mutant version of SV40 large T antigen; IntC: Cterminal intein; IntN: N-terminal intein; NCre: N-terminal Cre fragment; PV: Parvalbumin; RNA: Ribonucleic acid; RV-G: Rabies virus glycoprotein; SST: Somatostatin; VSV-G: Vesicular stomatitis virus glycoprotein

\section{Acknowledgments}

We would like to thank Drs. Henry Yin, Qing Yan, and George Yu for experimental assistance. We would like to also thank Drs. Wang Linfa and Danielle Anderson for their advice and help with viral constructs. The authors acknowledge the facilities, and the scientific and technical assistance of the Advanced Bioimaging Core, DUKE-NUS Singapore Health Services.

\section{Authors' contributions}

A.K. and H.S.J. constructed plasmids. H.K. generated the 3D model of reconstituted split-intein mediated split-Cre recombinase. H.S.J. performed western blot analyses. A.K. designed and performed HEK293T reporter assays, in vivo stereotaxic injections, genotyping and histological analyses. P.K. performed in utero electroporation. H.S.J. supervised the project. A.K. and H.S.J. contributed to ideas and designed and wrote the manuscript. The author(s) read and approved the final manuscript.

\section{Funding}

This research was supported by the Ministry of Education, Singapore, under its Academic Research Fund (MOE2014-T2-2-071), Singapore Ministry of Health's National Medical Research Council under its Individual Research Grant (NMRC/OFIRG/0050/2017 and NMRC/TCR/013-NNI/2014), National Research Foundation under its National Research Foundation Grant (NRF/ CRP17-2017-04) and Duke-NUS Signature Research Programme funded by the Ministry of Health Singapore under its Duke-NUS SRP Phase 2 Research Block Grant (all to H.S.J.)

Availability of data and materials Please contact author for data requests. 


\section{Ethics approval}

All animal procedures were approved by the Institutional Animal Care and Use Committee of Duke-NUS Medical School and SingHealth (IACUC \#2010/ SHS/590 and \#2014/SHS/921).

\section{Consent for publication}

All authors agreed to its submission to the Molecular Brain and, if accepted to its publication in this journal.

\section{Competing interests}

The authors declare no competing financial interests.

\section{Author details}

${ }^{1}$ Neuroscience and Behavioural Disorders Programme, Duke-National University of Singapore (NUS) Medical School, 8 College Road, Singapore 169857, Singapore. ${ }^{2}$ Graduate School of Medical Science \& Engineering, Korea Advanced Institute of Science and Technology (KAIST), Daejeon 34141, Republic of Korea. ${ }^{3}$ Center for Biomolecular \& Cellular Structure, Institute for Basic Science (IBS), Daejeon 34126, Republic of Korea.

Received: 7 May 2020 Accepted: 23 June 2020

Published online: 02 July 2020

\section{References}

1. de Lombares C, Heude E, Alfama G, Fontaine A, Hassouna R, Vernochet C, de Chaumont F, Olivo-Marin C, Ey E, Parnaudeau S, et al. Dlx5 and Dlx6 expression in GABAergic neurons controls behavior, metabolism, healthy aging and lifespan. Aging (Albany NY). 2019;11:6638-56.

2. del Valle Rodríguez A, Didiano D, Desplan C. Power tools for gene expression and clonal analysis in Drosophila. Nat Methods. 2012;9:47-55.

3. DeLano WL. PyMOL. DeLano scientific, San Carlos, CA, 700; 2002.

4. Dimidschstein J, Chen Q, Tremblay R, Rogers SL, Saldi GA, Guo L, Xu Q, Liu R, Lu C, Chu J, et al. A viral strategy for targeting and manipulating interneurons across vertebrate species. Nat Neurosci. 2016;19:1743-9.

5. Ennifar E, Meyer JEW, Buchholz F, Stewart AF, Suck D. Crystal structure of a wild-type Cre recombinase-loxP synapse reveals a novel spacer conformation suggesting an alternative mechanism for DNA cleavage activation. Nucleic Acids Res. 2003;31:5449-60.

6. Hirrlinger J, Scheller A, Hirrlinger PG, Kellert B, Tang W, Wehr MC, Goebbels S, Reichenbach A, Sprengel R, Rossner MJ, et al. Split-cre complementation indicates coincident activity of different genes in vivo. PLoS One. 2009;4: e4286.

7. Ho J, Tumkaya T, Aryal S, Choi H, Claridge-Chang A. Moving beyond P values: data analysis with estimation graphics. Nat Methods. 2019;16:565-6.

8. Jinno S, Klausberger T, Marton LF, Dalezios Y, Roberts JD, Fuentealba P, Bushong EA, Henze D, Buzsaki G, Somogyi P. Neuronal diversity in GABAergic long-range projections from the hippocampus. J Neurosci. 2007; 27:8790-804.

9. Jullien N, Goddard I, Selmi-Ruby S, Fina J-L, Cremer H, Herman J-P. Conditional transgenesis using Dimerizable Cre (DiCre). PLoS One. 2007;2: e1355.

10. Jullien N, Sampieri F, Enjalbert A, Herman JP. Regulation of Cre recombinase by ligand-induced complementation of inactive fragments. Nucleic Acids Res. 2003;31:e131.

11. Kaczmarczyk SJ, Green JE. Induction of cre recombinase activity using modified androgen receptor ligand binding domains: a sensitive assay for ligand-receptor interactions. Nucleic Acids Res. 2003;31:e86.

12. Kato S, Kobayashi K, Kobayashi K. Improved transduction efficiency of a lentiviral vector for neuron-specific retrograde gene transfer by optimizing the junction of fusion envelope glycoprotein. J Neurosci Methods. 2014;227: 151-8.

13. Lake BB, Ai R, Kaeser GE, Salathia NS, Yung YC, Liu R, Wildberg A, Gao D, Fung $\mathrm{H}$, Chen S, Vijayaraghavan R, Wong J, Cheng A, Sheng X, Kaper F, Shen R, Ronaghi M, Fan J, Wang W, Chun J, Zhang K. Neuronal subtypes and diversity revealed by single-nucleus RNA sequencing of the human brain. Science. 2016:352:1586-90

14. Le TN, Zhou Q-P, Cobos I, Zhang S, Zagozewski J, Japoni S, Vriend J, Parkinson T, Du G, Rubenstein JL, et al. GABAergic interneuron differentiation in the basal forebrain is mediated through direct regulation of glutamic acid decarboxylase isoforms by D/x homeobox transcription factors. J Neurosci. 2017;37:8816.
15. Lee AT, Vogt D, Rubenstein JL, Sohal VS. A class of GABAergic neurons in the prefrontal cortex sends long-range projections to the nucleus accumbens and elicits acute avoidance behavior. J Neurosci. 2014;34:1151925.

16. Lingawi NW, Balleine BW. Amygdala central nucleus interacts with dorsolateral striatum to regulate the acquisition of habits. J Neurosci. 2012; 32:1073-81.

17. Lizarraga SB, Coser KR, Sabbagh M, Morrow EM. Methods for study of neuronal morphogenesis: ex vivo RNAi electroporation in embryonic murine cerebral cortex. J Vis Exp. 2012;(63):e3621.

18. Lockless SW, Muir TW. Traceless protein splicing utilizing evolved split inteins. Proc Natl Acad Sci U S A. 2009;106:10999-1004

19. Madisen L, Zwingman TA, Sunkin SM, Oh SW, Zariwala HA, Gu H, Ng LL, Palmiter RD, Hawrylycz MJ, Jones AR, et al. A robust and high-throughput Cre reporting and characterization system for the whole mouse brain. Nat Neurosci. 2010;13:133-40.

20. Margolis EB, Lock H, Chefer VI, Shippenberg TS, Hjelmstad GO, Fields HL Kappa opioids selectively control dopaminergic neurons projecting to the prefrontal cortex. Proc Natl Acad Sci U S A. 2006;103:2938-42.

21. Mclntosh AR. Towards a network theory of cognition. Neural Netw. 2000;13: 861-70.

22. Melzer S, Gil M, Koser DE, Michael M, Huang KW, Monyer H. Distinct Corticostriatal GABAergic neurons modulate striatal output neurons and motor activity. Cell Rep. 2017:19:1045-55.

23. Nathanson $J$, Jappelli R, Scheeff ED, Manning G, Obata K, Brenner S, Callaway EM. Short promoters in viral vectors drive selective expression in mammalian inhibitory neurons, but do not restrict activity to specific inhibitory cell-types. Front Neural Circuits. 2009;3:19.

24. Perler FB. InBase: the intein database. Nucleic Acids Res. 2002;30:383-4.

25. Schultz W. Multiple reward signals in the brain. Nat Rev Neurosci. 2000;1: 199-207.

26. Shah NH, Muir TW. Inteins: Nature's gift to protein chemists. Chem Sci. 2014 5:446-61.

27. Shah NH, Muir TW. Split inteins: nature's protein ligases. Israel J Chem. 2011; 51:854-61.

28. Song AJ, Palmiter RD. Detecting and avoiding problems when using the Cre-lox system. Trends Genet. 2018:34:333-40.

29. Sporns O. Structure and function of complex brain networks. Dialogues Clin Neurosci. 2013;15:247-62

30. Tamamaki N, Tomioka R. Long-range GABAergic connections distributed throughout the Neocortex and their possible function. Front Neurosci. 2010; 4:202.

31. Tsien JZ. Cre-lox neurogenetics: 20 years of versatile applications in brain research and counting. Front Genetics. 2016;7:19.

32. Vercelli A, Repici M, Garbossa D, Grimaldi A. Recent techniques for tracing pathways in the central nervous system of developing and adult mammals. Brain Res Bull. 2000;51:11-28.

33. Wang B, Lufkin T, Rubenstein JLR. Dlx6 regulates molecular properties of the striatum and central nucleus of the amygdala. J Comp Neurol. 2011;519: 2320-34.

34. Wyart C, Del Bene F, Warp E, Scott EK, Trauner D, Baier H, Isacoff EY. Optogenetic dissection of a behavioural module in the vertebrate spinal cord. Nature. 2009:461:407-10.

35. Yuste R. From the neuron doctrine to neural networks. Nat Rev Neurosci. 2015:16:487-97.

36. Zhang W, Kim PJ, Chen Z, Lokman H, Qiu L, Zhang K, Rozen SG, Tan EK, Je HS, Zeng L. MiRNA-128 regulates the proliferation and neurogenesis of neural precursors by targeting PCM1 in the developing cortex. eLife. 2016;5: e11324.

37. Zheng $Y$, Wu Q, Wang C, Xu M-Q, Liu Y. Mutual synergistic protein folding in split intein. Biosci Rep. 2012:32:433-42.

\section{Publisher's Note}

Springer Nature remains neutral with regard to jurisdictional claims in published maps and institutional affiliations. 